\title{
On Cost Allocation in Networks With Threshold Based Discounting
}

\author{
Darko Skorin-Kapov \\ School of Business, Adelphi University, Garden City, New York
}

\begin{abstract}
We study network design in which each pair of nodes can communicate via a direct link and the communication flow can be delivered through any path in the network. The cost of flow through each link is discounted if and only if the amount of flow exceeds certain threshold. This exploitation of economies of scale encourages the concentration of flows and use of relatively small number of links. Applications include telecommunications, airline traffic flow, and mail delivery networks. The cost of services delivered through such a network is distributed among its users who may be individuals or organizations with possibly conflicting interests. The cooperation between these users is essential for the exploitation of economies of scale. Consequently, there is a need to ensure a fair distribution of the cost of providing the service among network users. In order to describe this cost allocation problem we formulate the associated cooperative game, to be referred to as the threshold game. We then demonstrate that certain cost allocation solution (the core of the threshold game) can be efficiently applied to relatively 'large' networks with threshold-based discounting.
\end{abstract}

Keywords: network design, cost allocation, cooperative games, hub-like networks

\section{Introduction}

The motivation for this work came from the studies of hub networks, which play an important role in modeling transportation and telecommunication systems. In hub communication networks a certain subset of focal nodes (i.e. hubs) is fully interconnected, while other nodes are connected to those hubs. Communication between the nodes is done exclusively via hubs. The assumption is that hub nodes are completely interconnected, while non-hub nodes are connected to one or more hubs. Moreover, the cost of traffic between hubs is discounted. The advantage of hub networks is the usage of relatively small number of links and exploitation of economies of scale by concentrating flows. The hub networks were extensively studied over the last couple of decades (see for example, $[1],[5],[6],[16]$ and [17]). Numerous computational studies show that hub networks are quite attractive and practical. Nevertheless, the restrictions imposed with the hub network model are sometimes too prohibitive. For example, in some cases, high traffic between a non-hub and a hub node is not discounted and/or the traffic between two hubs is not big enough to warrant any discounts. For an extensive discussion on these issues see [7].

Recently, Podnar et al. [7] introduced the network model in which each pair of nodes can communicate via any path, and the cost of sending flow through each link is discounted if and only if the amount of flow exceeds certain threshold. This approach also gives incentive to concentrate flows. It seems however, that the above threshold-based discounting model is even more 'efficient' than hub networks in its use of a relatively small number of links and in the exploitation of economies of scale. We will refer to the threshold-based discounting network model as to the hub-like network (HLN) model. Their paper provides combinatorial formulations and efficient heuristic for finding the minimum cost HLN.

The cost of services delivered through a hublike network is distributed among its users who may be individuals or organizations with possibly conflicting interests. The cooperation between these users is essential for the exploitation of economies of scale. Consequently, there is a need to ensure a fair distribution of the cost of providing the service among users of a 
hub-like network. Failing to do so may cause some users to secede and seek services from some other, competing network. Such secession would inevitably result in a higher cost per unit of delivered service.

A cooperative game theory was used to analyze several classes of network cost allocation problems in the literature. Some examples include: spanning tree games ([4]), Steiner tree games ([11]), network flow games $([2],[3])$, cost allocation arising from routing in networks ([8]), capacitated network design games $([10],[15])$, hub network games $([12])$. For a survey and numerous references on cost allocation models in networks see, [9]. A common approach to above papers is the formulation of the associated cost allocation problem as a cooperative game in characteristic function form, followed by the evaluation of various solution concepts such as core, nucleolus, kernel, the least $\epsilon$-core, Shapley value, etc. It is well known that these game theoretic solution concepts are computationally prohibitive even for relatively small problems. Moreover, there are no general practical algorithms for the computation of these solutions. Consequently, researchers have concentrated on individual classes of games to demonstrate that computation of cost allocation solution concepts is sometimes feasible in the context of a particular problem.

The cost allocation problem associated with the HLN was first introduced by D. Skorin-Kapov $[13,14]$. Therein, the hub-like game which emphasizes the users' contribution to economies of scale was defined. In addition, the core of the hub-like game was efficiently characterized (by $\left(\mathcal{O}\left(n^{4}\right)\right.$ constraints). This characterization is associated with the tight 4-dimensional formulation of the HLN model presented in [7]. Namely, the best known solution obtained by the 4-dimensional formulation of the HLN problem is used as an input to the associated cost allocation problem. Note however, that the HLN optimization model becomes too large to handle with 4-dimensional formulation even for networks with 20 nodes.

In this paper, we will revisit the above cost allocation problem. The main contribution of this paper is that it gives us the game theoretic cost allocation solution, which is computationally tractable for some larger HLN problems $(20,25$ and even more nodes). Indeed, we will formulate yet another associated cooperative game, to be referred to as the threshold game. We then demonstrate that the core of the threshold game can be characterized by $\left(\mathcal{O}\left(n^{4}\right)\right)$ constraints. This characterization of the core has the same computational complexity as the characterization in [13 and 14]. Nevertheless, there are profound differences. Namely, the players in the threshold game are users (nodes) rather than pairs of users. Moreover, the definition of the threshold game is based on the 3-dimensional formulation of the HLN problem given in [7]. This in turn enables an efficient characterization of the core of the threshold game which is applicable for some larger HLN problems.

The plan of the reminder of the paper follows. In Section 2, the HLN problem and some game theoretic concepts are defined. In Section 3, we formulate the threshold game associated with the HLN problem. Section 4 provides the analysis of the core of the threshold game. In Section 5, our findings and concluding remarks are summarized.

\section{Definitions and Preliminaries}

For completeness, we re-state the 4- and 3dimensional formulations of the HLN problem from [7]. The decision variables are given as follows. Let $N$ be the set of nodes. The cost of sending the unit of flow is assigned to every link by cost matrix $D=\left(d_{k m}\right)$. Input matrix $F=\left(f_{i j}\right)$ contains the required amounts of flow associated with every origin-destination pair $(i, j)$. Variable $x 1_{k m}^{i j}$ captures the fraction of flow that goes from node $i$ to node $j$ via link $(k, m)$ which is not discounted. Variable $x 2_{k m}^{i j}$ is the fraction of flow from $i$ to $j(\operatorname{via}(k, m)$ ) that is discounted. Parameter $\alpha, 0<\alpha<1$ is the discount factor. Binary variable $y_{k m}$ is 1 if link $(k, m)$ is discounted and 0 otherwise. The HLN problem is then:

$$
\min \sum_{i, j, k, m, i \neq j, k \neq m, m \neq i, k \neq j} d_{k m}\left(x 1_{k m}^{i j}+\alpha x 2_{k m}^{i j}\right)
$$


s.t.

$$
\begin{gathered}
\sum_{\substack{i, j, i \neq j, i \neq m, j \neq k \\
\text { for all } k, m}} x 2_{k m}^{i j} \geq y_{k m} Q, \\
x 2_{k m}^{i j} \leq y_{k m} f_{i j},
\end{gathered}
$$

for all $i, j, k, m: i \neq j, k \neq m, m \neq i, k \neq j$

$$
\sum_{m: m \neq i}\left(x 1_{i m}^{i j}+x 2_{i m}^{i j}\right)=f_{i j}
$$

$$
\text { for all } i, j: i \neq j
$$

$$
\begin{gathered}
\sum_{k: k \neq l, k \neq j}\left(x 1_{k l}^{i j}+x 2_{k l}^{i j}\right)=\sum_{m: m \neq l, m \neq i}\left(x 1_{m l}^{i j}+x 2_{m l}^{i j}\right) \\
\text { for all } l, i, j: l \neq i, j, i \neq j \\
x 1, x 2 \geq 0, y \text {-binary. }
\end{gathered}
$$

If the flow through a link $(k, m)$ is discounted, then it must be greater than the threshold $Q$ (constraints $(2))$. In the case when the link $(k, m)$ is not discounted, the variables $x 2_{\mathrm{km}}^{i j}$ are set to zero (constraint (3)). The complete flow $f_{i, j}$ must leave the origin $i$ (constraint (4)). Conservation of flow follows from constraint (5). Nonnegativity and integrality are ensured by constraints (6).

The complexity of this model is $\left(\mathcal{O}\left(n^{4}\right)\right)$. Computational studies [7] showed that the LP relaxation of this model is very tight. However, for larger problems, the size of the problem becomes restrictive. Airline transportation and telecommunication networks are often symmetric. Hence, it made sense to consider a symmetric case $\left(d_{k m}=d_{m k}, f_{i j}=f_{j i}\right)$ and also require symmetry of the solution (i.e. $x 1_{k m}^{i j}=x 1_{m k}^{j i}$, $x 2_{k m}^{i j}=x 2_{m k}^{j i}$ and $\left.y_{k m}=y_{m k}\right)$. This reduced the number of variables by half, but the dimension of the model remained $\left(\mathcal{O}\left(n^{4}\right)\right)$.

It appears (see [7]) that the 4-dimensional formulation of the HLN model is restrictive with respect to the size of the problem. For that reason, we will also consider the 3-dimensional formulation. Since we are interested in the amount of flow that goes through each link $(k, m)$, we will ignore the destination of the flow. Disregarding the destinations we define variables as follows:

$x 1_{k m}^{i}=$ non-discounted flow originated from $i$ through link $(k, m)$,

$x 2_{k m}^{i}=$ discounted flow originated from $i$ through link $(k, m)$,

$y$ is defined as in the above 4-dimensional problem, where

$$
x 1_{k m}^{i}=\sum_{j: j \neq i, k} x 1_{k m}^{i j}, x 2_{k m}^{i}=\sum_{j: j \neq i, k} x 1_{k m}^{i j} .
$$

The $\left(\mathcal{O}\left(n^{3}\right)\right)$ formulation of the HLN problem is then:

$$
\min \sum_{i, k, m, k \neq m, m \neq i} d_{k m}\left(x 1_{k m}^{i}+\alpha x 2_{k m}^{i}\right)
$$

s.t.

$$
\sum_{i, i \neq m} x 2_{k m}^{i} \geq y_{k m} Q
$$

for all $k, m: k \neq m$

$$
x 2_{k m}^{i} \leq y_{k m} \sum_{j: j \neq i, j \neq k} f_{i j},
$$

for all $i, k, m: k \neq m, m \neq i$

$$
\begin{gathered}
\sum_{k: k \neq l}\left(x 1_{k l}^{i}+x 2_{k l}^{i}\right)-\sum_{m: m \neq l, m \neq i}\left(x 1_{m l}^{i}+x 2_{m l}^{i}\right)=f_{i l}, \\
\text { for all } l, i: l \neq i \\
x 1, x 2 \geq 0, y \text {-binary. }
\end{gathered}
$$

Constraints (8), (9) and (11) have the same role as in the 4-dimensional case. Constraint (10) implies that the difference between the flow coming into $l$ and the flow going out of $l$ is equal to $f_{i l}$.

The loss of information in the 3-dimensional case due to ignoring of destinations results with the larger feasible set (i.e. the LP relaxation is not as tight as in the 4-dimensional case). On the other hand, the advantage is, that it can handle larger problems. In [7], the authors presented several heuristic algorithms to solve the HLN problem. Moreover, they performed extensive computational experiments on the $\mathrm{CAB}$ (Civil Aeronautics Board) benchmark data set. 
The efficiency of their formulations and heuristic was demonstrated by obtaining the gaps between upper and lower bounds within few percent.

In order to analyze the cost allocation problem associated with the HLN problem, we need to introduce the following game theoretic definitions and notation. Let $P=\{1,2, \ldots, n\}$ be a finite set of players, and let $c: 2^{P} \rightarrow \mathbf{R}$, with $c(\emptyset)=0$, be a characteristic function defined over subsets of $P$ referred to as coalitions. If $\mathrm{c}(P)$ designates a cost that has to be shared by all the players, then the pair $(P ; \mathrm{c})$ is called a (cost) cooperative game, or simply a game. For $x \in \mathbf{R}^{|P|}$ and $S \subseteq P$, let $x(S) \equiv \sum_{j \in S} x_{j}$. We can interpret $x(S)$ as the part of the total cost paid by the coalition $S$. A cost allocation vector $x$ in a game $(P ; c)$ satisfies $x(P)=c(P)$, and the solution theory of cooperative games is concerned with the selection of a reasonable subset of cost allocation vectors.

Central to the solution theory of cooperative games is the concept of solution referred to as the core of a game. The core of a game $(P, c)$ consists of all vectors $x \in \mathbf{R}^{|P|}$ such that $x(S) \leq c(S)$ for all $S \subseteq P$, and $x(P)=c(P)$. Observe that the core consists of all allocation vectors $x$ which provide no incentive for any coalition to secede.

\section{The Threshold Game}

In this section, we will use a game theoretic approach to describe the cost allocation problem in networks with threshold-based discounting. Specifically, we will formulate associated cooperative threshold game. The total cost of delivering the service through a hub-like network is obtained from the best known objective function value to the 3-dimensional HLN problem $((7)-(11))$. The objective is to allocate this cost among network users in a 'fair' manner.

In order to define a threshold game we first have to identify the players. The natural choice for the set of players seem to be the set of all nodes, namely $P=N$. Observe that in [13 and 14] the players were pairs of nodes. Since we use here a 3-dimensional formulation of the HLN problem as input to our cost allocation problem, we know for each link the amount of flow and the source where it came from, but we do not know the exact destination of the flow. Consequently, the use of pairs of nodes for players is not applicable. Moreover, since we do not know the destinations of flows in our solution, the above choice of players (i.e. $P=N$ ) is appropriate only for symmetric problems.

Next, we will define the characteristic function $c$. For an empty set $c(\emptyset)=0$. For $\emptyset \neq T \subseteq N$, we first define the cost of flow generated by nodes in $T$ through each link. Let $c_{k m}(T)$ be the cost of flow that players in a coalition $T$ send through a link $(k, m)$ in the optimal (best known) hub-like network. Note that we assume here that the coalition $T$ uses the same links and costs as in a globally optimal solution. The difference is that the cost of the above portion of flow originated from nodes in $T$ through $(k, m)$, is discounted if and only if $T$ itself generates enough traffic for the discount (the amount exceeds the threshold). For each link $(k, m)$, we call $\left(N, c_{k m}\right)$ the link game. For each $T \subseteq N$, let $c(T)=\sum_{(k, m)} c_{k m}(T)$. Game $(N, c)$ will be called the threshold discounting network game, or, for short, the threshold game.

\section{Core of the Threshold Game}

Recall that the core consists of all cost allocation vectors that provide no incentive for any coalition to secede. Specifically, each cost allocation vector in the core of a threshold game allocates to each coalition of players at most the cost needed to provide service to that coalition. Namely, there is no cross-subsidization. In this Section, we will show that the core of a threshold game can be efficiently characterized.

\section{Decomposition Theorems}

We will demonstrate that the threshold game can be decomposed into link games. Namely, in order to analyze the core of the threshold game it is sufficient to consider all corresponding link games.

Theorem 1. For each link $(k, m)$, let $x_{k m}$ be a point in the core $\mathcal{C}\left(N, c_{k m}\right)$ of the link game $\left(N, c_{k m}\right)$. Then, $x=\sum_{(k, m)} x_{k m}$ is in the core $\mathcal{C}(N, c)$ of the threshold game $(N, c)$. 
Proof. Since $x_{k m} \in \mathcal{C}\left(N, c_{k m}\right)$ for all links $(k, m), x_{k m}(T) \leq c_{k m}(T)$ for all links $(k, m)$ and all coalitions $T \subseteq N$. This implies that $\sum_{(k, m)} x_{k m}(T) \leq \sum_{(k, m)} c_{k m}(T)$, and then $x(T)$ $\leq c(T)$ for all $T \subseteq N$.

Theorem 2. Let $x$ be a cost allocation in the threshold game. Define allocations for the related link games as follows. For each link $(k, m)$ and a player $i \in T$, let the portion of the cost covered by the player $i$ be $x_{k m}(i)=x(i) \frac{c_{k m}(i)}{c(i)}$. If $x$ is in the core $\mathcal{C}(N, c)$ of the threshold game then for each link $(k, m), x_{k m}$ is in the core $\mathcal{C}\left(N, c_{k m}\right)$ of the related link game.

Proof. For any link $(k, m)$ and any coalition $T \subseteq N$ :

$$
\begin{aligned}
x_{k m}(T) & =\sum_{i \in T} x_{k m}(i)=\sum_{i \in T} x(i) \frac{c_{k m}(i)}{c(i)} \\
\leq & \sum_{i \in T} c_{k m}(i)=c_{k m}(T) .
\end{aligned}
$$

The above inequality holds since the assumption $x \in \mathcal{C}(N, c)$ implies that $x(i) \leq c(i)$, for all $i \in T$. Hence, for each link $(k, m), x_{k m}$ is in $\mathcal{C}\left(N, c_{k m}\right)$.

Corollary 1. In order to characterize the core $\mathcal{C}(N, c)$ of the threshold game $(N, c)$, it is sufficient to characterize the cores $\mathcal{C}\left(N, c_{k m}\right)$ of link games $\left(N, c_{k m}\right)$, where $(k, m)$ are links in the optimal hub-like network.

\section{Characterization Theorems}

In general, the main computational difficulty with the core is that it is characterized with an exponential number of constraints. Nevertheless, we will show that most of the core constraints for the core of each link game are redundant. Namely, we will characterize the core of the link game with $\left(\mathcal{O}\left(n^{2}\right)\right)$ constraints.

Lemma 1. Consider a link game $\left(N, c_{k m}\right)$. Let $T \subseteq N$, be a coalition which generates enough traffic to warrant the discount $\alpha$ on the traffic through link $(k, m)$, and let $x_{k m}$ be the cost allocation which satisfies the core constraints associated with coalitions $N \backslash\{i\}$, for all $i \in N \backslash T$. Then, for each $i \in N \backslash T$ :

$c_{k m}(T)-x_{k m}(T) \geq c_{k m}(T \cup\{i\})-x_{k m}(T \cup\{i\})$.
Proof. Since the traffic generated by $T$ is eligible for the discount on the link $(k, m)$, so is the traffic generated by any superset of $T$. Then, by definition of the characteristic function $c_{k m}$, for each $i \in N \backslash T$ we have:

$$
\begin{aligned}
& c_{k m}(N)-c_{k m}(N \backslash\{i\}) \\
& =c_{k m}(T \cup\{i\})-c_{k m}(T) .
\end{aligned}
$$

On the other hand,

$$
c_{k m}(N \backslash\{i\})-x_{k m}(N \backslash\{i\}) \geq 0
$$

implies that

$$
c_{k m}(N \backslash\{i\})-x_{k m}(N)+x_{k m}(i) \geq 0,
$$

and then

$$
x_{k m}(i) \geq c_{k m}(N)-c_{k m}(N \backslash\{i\}) .
$$

Now from (12) and (13) it follows that

$$
x_{k m}(i) \geq c_{k m}(T \cup\{i\})-c_{k m}(T),
$$

which further implies that

$c_{k m}(T)-x_{k m}(T) \geq c_{k m}(T \cup\{i\})-x(T \cup\{i\})$.

Let $f_{k m}^{i}$ be the amount of flow that the user $i$ generates through a link $(k, m)$. Let the collection $\mathcal{S}_{1}=\left\{\{i\} \mid i \in N\right.$ and $\left.f_{k m}^{i}<Q\right\}$ consist of all single player coalitions which themselves do not generate sufficient amount of traffic through the link $(k, m)$ to warrant the discount $\alpha$, and let $\mathcal{S}_{2}=\{N \backslash\{i\} \mid i \in N\}$ be the collection of all coalitions that are missing only a single player.

Theorem 3. The core constraints associated with coalitions in collections $\mathcal{S}_{1}$ and $\mathcal{S}_{2}$ completely determine the core of the link game $\left(N, c_{k m}\right)$.

Proof. Assume that the core constraints associated with all coalitions belonging to collections $\mathcal{S}_{1}$ and $\mathcal{S}_{2}$ are satisfied. Let the coalition $T \subset N$ be such that $T \notin \mathcal{S}_{1}$ or $\mathcal{S}_{2}$. We need to consider two cases. 
(i) If the total traffic generated by users in $T$ is not sufficient to enable the discount for the traffic on the link $(k, m)$, then each user in $T$ is a single player coalition belonging to $\mathcal{S}_{1}$, and:

$$
\begin{gathered}
c_{k m}(T)-x_{k m}(T)=\sum_{i \in T}\left(c_{k m}(i)-x_{k m}(i)\right) \\
\geq c_{k m}(i)-x_{k m}(i) \geq 0 .
\end{gathered}
$$

(ii) If the total traffic generated by $T$ is sufficient for the discount on the link $(k, m)$, then for any $i \in N \backslash T$, by the inductive application of Lemma 4 it follows that:

$$
\begin{aligned}
& c_{k m}(T)-x_{k m}(T) \\
& \geq c_{k m}(N \backslash\{i\})-x_{k m}(N \backslash\{i\}) \geq 0 .
\end{aligned}
$$

Hence, the core condition associated with the coalition $T$ is redundant.

\section{Conclusions}

In this paper, we investigated the cost allocation problem associated with the threshold-based discounting (hub-like) network design problem. The objective was to develop a procedure to allocate the cost in a fair manner using cooperative game theory approach. By fair, we mean that each subset of users should be charged a share of the network cost which provide them no incentive to seek services from another network. There are no definite answers to the issue of fairness, but the theory of cooperative games proposes several solution concepts. To that end we first formulated cooperative game referred to as the threshold game.

Our model emphasizes the users' contribution to economies of scale. We demonstrated that some cost allocation solution(s) associated with these games can be efficiently computed. Specifically, the threshold game $(N, c)$ can be decomposed into $n^{2}$ link games $\left(N, c_{k m}\right)$, and the core of each link game can be characterized with $2 n$ linear constraints (T.3). Hence, the core of the threshold game $(N, c)$ can be characterized with $\mathcal{O}\left(n^{3}\right)$ constraints.

The input to our cost allocation problem is the best known solution to the symmetrical hub-like network problem. Note that as input we can take the solution to either 4- or 3-dimensional version of the problem. Specifically, using these inputs we can generate the relevant constraints (T.3) for corresponding link games. Then, every collection of feasible solutions to cores of link games would give us a core point of the threshold game (T.1), and all such collections would give us the entire core of the threshold game (T.2).

In summary, we developed a framework for the efficient computation of some cost allocation solutions in hub-like network. In those solutions users are charged a fair share of the cost with respect to their contribution to economies of scale. Such a scheme gives the users incentive to cooperate and exploit economies of scale via participation in a threshold-based discounting network.

\section{References}

[1] CAmpBell, J.F., (1994b), "A Survey of Network Hub Location," Studies in Locational Analysis, Issue 6, 31-49.

[2] Granot, D. And F. GRanot, "On Some Network Flow Games", Mathematics of Operations Research, Vol. 17, No.4, (1992) 792-841.

[3] Granot, D. AND M. Hojati, "On the Cost Allocation in Telecommunication Networks", Networks, Vol. 20 (1990) 209-229.

[4] Granot, D. and G. Huberman, "Minimum Cost Spanning Tree Games," Math. Programming 21 (1981) 1-18.

[5] O’Kelly, M.E., “A Quadratic Integer Program for the Location of Interacting Hub Facilities," European Journal of Operational Research, 32, (1987) 393-404.

[6] O'Kelly, M., D. SKorin-Kapov, AND J. SKORINKAPOV, "Lower Bounds for the Hub Location Problem", Management Science, Vol. 41, No. 4, (1995) 713-728.

[7] PODNAR, H., J. SKORIN-KAPOV AND D. SKORINKAPOV, "Network Cost Minimization Using Threshold-Based Discounting", Technical Paper, SUNY at Stony Brook, Applied Mathematics and Statistics, (October 1999).

[8] POTTERS, J.A.M., I.J. CURIEL AND S.H. TIJS, “Traveling Salesman Games", Mathematical Programming 53 (1987) 199-211.

[9] SHARKEY, W. W., "Networks Models in Economics", Chapter 9 in $M$. O. Ball et. al. Eds., Handbooks in OR\&MS, Vol. 8, Elsevier (1995). 
[10] SKORIN-KAPOV, D., "On a Cost Allocation Problem Arising From a Capacitated Concentrator Covering Problem", Operations Research Letters, Vol. 13, No. 5, (1993) 315-323.

[11] SkORIN-Kapov, D., "On the Core of the Minimum Steiner Tree Game in Networks", Annals of Operations Research, 57 (1995) 233-249.

[12] SKORIN-KAPOV, D., Hub Network Games, Networks 31, (1998) 293-302

[13] SKORIN-KAPOV, D., On Cost Allocation in Hub Like Networks, Working Paper, Adelphi University, September 2000.

[14] SKORIN-KAPOV, D., On Cost Allocation in Networks With Threshold Based Discounting, Proceedings of the 22nd International Conference on Information Technology Interfaces, ITI'2000, Pula (2000) 409-414.

[15] SKORIN-KAPOV, D. AND H.F. BELTRAN, "An Efficient Characterization of Cost Allocation Solutions Associated With Capacitated Network Design Problems", Telecommunication Systems, Vol. 3, No. 1, (1994) 91-107.

[16] SKOrIN-KAPOV, D. AND J. SKORIN-KAPOV, (1994), "On Tabu Search for the Location of Interacting Hub Facilities", European Journal of Operational Research. 73(1994)502-509.

[17] SKORIN-KAPOV, D., J. SKORIN-KAPOV AND M. O’Kelly, (1995), “Tight Linear Programming Relaxations of Uncapacitated p-hub Median Problems", European Journal of Operational Research 94 (1996) 582-593.

Received: October, 2000 Accepted: November, 2000

Contact address:

Darko Skorin-Kapov School of Business Adelphi University Garden City, NY 11530

e-mail: skorin@adelphi.edu

DARKO SKORIN-KAPOV is an Associate Professor of Information Technology in the School of Business, Adelphi University, Garden City, New York. He got his B. Sc. and M. Sc. in Mathematics and Information Science from the University of Zagreb, Croatia. He received his $\mathrm{Ph}$. D. in Management Science from the University of British Columbia, Vancouver, B. C., Canada. His research is predominantly in the area of network optimization and cost allocation. He has extensive academic, as well as, industrial experience in those areas. His publications include papers in Annals of OR, Discrete Applied Mathematics, European Journal of OR, Location Science, Management Science, Networks, OR Letters, SIAM Journal of Discrete Mathematics and Telecommunication Systems. 